\title{
Excessive Daytime Sleepiness and Cardiovascular Mortality in US Adults: A NHANES 2005-2008 Follow-Up Study
}

\author{
Jingen Li (iD) ${ }^{1,2}$ \\ Naima Covassin ${ }^{2}$ \\ Joshua M Bock ${ }^{2}$ \\ Essa A Mohamed ${ }^{2}$ \\ Lakshmi P Pappoppula ${ }^{2}$ \\ Chilsia Shafi ${ }^{2}$ \\ Francisco Lopez-Jimenez ${ }^{2}$ \\ Virend K Somers ${ }^{2}$ \\ 'Department of Cardiovascular Medicine, \\ Dongzhimen Hospital, Beijing University \\ of Chinese Medicine, Beijing, 100700, \\ People's Republic of China; ${ }^{2}$ Department \\ of Cardiovascular Medicine, Mayo Clinic, \\ Rochester, MN, 55902, USA
}

Correspondence: Virend K Somers Department of Cardiovascular Medicine, Mayo Clinic, Rochester, MN, 55902, USA Tel + I 507 28425 I I

Email somers.virend@mayo.edu
Purpose: Excessive daytime sleepiness is highly prevalent and has been associated with increased risk of cardiovascular diseases, but evidence for its association with cardiovascular mortality is limited and inconsistent. We aimed to determine whether excessive daytime sleepiness is independently associated with cardiovascular mortality in general adult population.

Patients and Methods: A prospective study of 10,330 adult participants (aged $\geq 20$ years) from National Health and Nutrition Examination Survey (NHANES) 2005-2006 and 20072008 was followed up until December 31st, 2015. Excessive daytime sleepiness was defined as the self-reported feeling of being overly sleepy often or always during the day. Cox proportional hazard ratios (HRs) with $95 \%$ confidence interval (CI) were estimated to assess risk for cardiovascular mortality.

Results: A total of 10,330 participants with mean age of 47.3 years (95\% CI, 46.0 to 48.1 ) were included in this analysis. Approximately, $18.5 \%$ of US adults reported excessive daytime sleepiness. Over a mean follow-up of 8.3 years, 262 cardiovascular deaths occurred. Participants with excessive daytime sleepiness had 2.85-times greater risk (95\% CI, 1.33 6.09) of cardiovascular death than those without daytime sleepiness in multivariable analysis corrected for sociodemographic factors, comorbidities and cardiovascular risk factors including depression. Further adjustment for self-reported sleep disorders and sleep duration only slightly attenuated this association (HR, 2.55; 95\% CI, 1.23-5.27). No interactions between excessive daytime sleepiness and age, sex or cardiovascular disease at study entry were observed (all $P s>0.05$ ).

Conclusion: Excessive daytime sleepiness is highly prevalent among US adults and is independently associated with an approximately two-and-a-half-fold increased risk of cardiovascular mortality in a large national sample. Screening for excessive daytime sleepiness may be a simple and cost-effective tool for identifying individuals at high risk of cardiovascular death.

Keywords: sleep disorders, cardiovascular diseases, cardiovascular risk, cohort study

\section{Introduction}

Excessive daytime sleepiness (EDS) indicates difficulty in maintaining a desired level of wakefulness and alertness at appropriate times during the day. It is estimated that 5$30 \%$ of the general population endorses complaints of EDS. ${ }^{1-4}$ Growing evidence suggests that EDS is associated with a broad range of clinical and non-clinical consequences, including increased risk of motor vehicle accidents, ${ }^{5}$ occupational injuries, ${ }^{6}$ and loss of productivity, ${ }^{7}$ along with cognitive impairment, ${ }^{8}$ reduced quality 
of life and increased socioeconomic burden. ${ }^{9}$ Associations between EDS and traditional cardiovascular risk factors, including obesity, hypertension, and diabetes, have also been reported in multiple studies. ${ }^{2,10}$ Although a few studies found no relationship between EDS and cardiovascular diseases, ${ }^{11,12}$ the majority of published reports showed significantly increased risk of prevalent and incident cardiovascular diseases in individuals with EDS. ${ }^{13-19}$ However, there is little evidence on the implications of EDS for cardiovascular mortality. To our knowledge, only a few studies assessed this relationship, yielding mixed results. EDS was a significant predictor of cardiovascular death in a community-dwelling cohort of 8269 older ( $\geq 65$ years) adults. ${ }^{20}$ Conversely, two other prospective populationbased studies on urban community residents $(\geq 40 \text { years })^{13}$ and a general population sample of men aged 30-69 years ${ }^{21}$ reported no significant association between EDS and cardiovascular death. Furthermore, it should be noted that none of these studies controlled for the potential role of sleep duration or comorbid sleep disorders and that no study has assessed the association of EDS and cardiovascular mortality in the general population including both males and females.

Therefore, we sought to clarify the association between EDS and cardiovascular mortality using the combined nationally representative data from the US National Health and Nutrition Examination Survey (NHANES) 2005-2006 and NHANES 2007-2008.

\section{Patients and Methods}

\section{Data Source}

NHANES comprises a series of ongoing, cross-sectional, nationally representative surveys of non-institutionalized US civilians. A stratified, multistage probability sampling method is applied to select participants. ${ }^{22}$ Data on demographics, socioeconomic status, health conditions, and health-related behaviors were collected during in-home interviews while physical measurements and laboratory data were obtained using mobile examination centers (MEC). Mortality data were acquired through linkage to the National Death Index. NHANES protocols were approved by the National Center for Health Statistics Ethics Review Board and written informed consent was obtained from all participants.

\section{Study Population}

Data from two NHANES cycles (ie, 2005-2006 and 20072008) were combined based on the questionnaire enabled collection of sleep disorders and symptoms in these cycles. A total of 10,914 adult participants were enrolled in NHANES 2005-2008. After excluding participants who were pregnant or uncertain of pregnancy status (women aged between 20 and 59 years not taking pregnancy test) $(\mathrm{n}=552)$, or those missing sleepiness $(\mathrm{n}=20)$ or mortality $(\mathrm{n}=12)$ data, 10,330 adult participants (aged $\geq 20$ years) were included in the final analysis (Figure A in supplement).

\section{Assessment of Excessive Daytime Sleepiness}

Data on daytime sleepiness were obtained during in-home interviews using a sleep questionnaire. Daytime sleepiness was determined by the question "In the past month, how often do you feel overly sleepy during the day?" with possible responses being "Never, Rarely (1 time a month), Sometimes (2-4 times a month), Often (5-15 times a month) and Almost Always (16-30 times a month)." We categorized participants into three groups according to the responses: never, rarely/sometimes, and often/always, which were, respectively, defined as no, mild and excessive daytime sleepiness consistent with a prior study. ${ }^{20}$

\section{Ascertainment of Cardiovascular Mortality}

Vital status was ascertained by linkage to the National Death Index through December 31, 2015. Details of the linking methods are described by the National Center for Health statistics. ${ }^{23}$ Causes of death were classified according to the International Statistical Classification of Diseases, 10th revision (ICD-10) codes. Cardiovascular mortality was defined as death caused by hypertensive heart disease (I11), hypertensive heart and renal diseases (I13), ischemic heart disease (I20-I25), acute rheumatic fever and chronic rheumatic heart diseases (I00-I09) and other heart diseases (I26-I51) including heart failure (I50) and atrial fibrillation (I48).

\section{Covariates}

Socioeconomic factors including age, sex, race (Hispanic, non-Hispanic white, non-Hispanic black and other), family income $(<\$ 20,000 /$ year or not), and education (ever attend college or not) were also obtained from in-home interview questionnaires. Sleep duration was determined by the question "How much sleep do you usually get at night 
on weekdays or workdays?" and we categorized the sleep duration into three groups ( $\leq 6 \mathrm{~h}, 7-8 \mathrm{~h}$ or $\geq 9 \mathrm{~h}$ ). Sleep disorders were ascertained by a positive response ('yes') to the questions "Have you ever been told by a doctor or other health professional that you have a sleep disorder?" and if the participant responded with 'yes', type of sleep disorders (sleep apnea, insomnia, restless legs and other) would be required. Cardiovascular risk factors and comorbidities were obtained from both in-home interview questionnaires and laboratory examination or tests from mobile examination centers: Alcohol consumption was determined by the question 'Have you ever had at least 12 alcohol drinks per year?'. Smoking was categorized into current ('Yes' to the question 'Do you now smoke cigarettes'), former ('Yes' to the question 'Have you smoked at least 100 cigarettes in your entire life' but 'No' to the question 'Do you now smoke cigarettes') and never ('No' to the question 'Have you smoked at least 100 cigarettes in your entire life'). Hypercholesterolemia was defined as self-reported history of hypercholesterolemia, serum cholesterol level $\geq 240 \mathrm{mg} / \mathrm{dL}$ [multiply by 0.02586 to get $\mathrm{mmol} / \mathrm{L}$ ] or being told to take prescriptions for cholesterol lowering, similar to the determination of hypertension (self-reported history of high blood pressure, mean blood pressure $\geq 140 / 90 \mathrm{mmHg}$ or being told to take prescribed medicine for high blood pressure), diabetes mellitus (selfreported history of diabetes, fasting glucose level $\geq 126 \mathrm{mg} / \mathrm{dL}$ [multiply by 0.0555 to get $\mathrm{mmol} / \mathrm{L}$ ], hemoglobin A1C $\geq 6.5 \%$ or now taking insulin or blood glucose lowering medication) and chronic kidney diseases (selfreported history of having weak or failing kidneys, glomerular filtration rate $[\mathrm{GFR}]<60 \mathrm{~mL} / \mathrm{min} / 1.73 \mathrm{~m}^{2}$ or albumin-to-creatinine ratio $>30 \mathrm{mg} / \mathrm{g}$ ). GFR was calculated using the Chronic Kidney Disease Epidemiology Collaboration (CKD-EPI) equation. ${ }^{24}$ Chronic obstructive pulmonary diseases (COPD, 'yes' to the question 'told by a doctor have emphysema or chronic bronchitis') and cancer ('Yes' to the question 'ever told by a doctor you had cancer or malignancy') were all determined by participants' self-reported medical history. Cardiovascular disease was determined by the reporting of any one of the following conditions: congestive heart failure, coronary heart disease, angina, heart attack and stroke. Depression was assessed using the Patient Health Questionnaire 9-item survey (PHQ-9, ranging from 0 to 27 with higher scores indicating higher levels of depressive symptoms), with a score $\geq 10$ indicative of major depression. ${ }^{25}$ Body mass index (BMI) was calculated by objectively measured weight $(\mathrm{kg})$ divided by height in meters squared $\left(\mathrm{m}^{2}\right)$.

\section{Statistical Analysis}

Complex survey design factors in NHANES, including sample weights, clustering, and stratification were all taken into account as recommended. ${ }^{26}$ Baseline characteristics were compared across daytime sleepiness categories using the Rao-Scott $\chi 2$ test for categorical variables and analysis of variance adjusted for sampling weights for continuous variables. ${ }^{27}$

Hazard ratios (HRs) with 95\% confidence interval (CI) were calculated by Cox proportional hazards models using SPSS 20.0 (IBM Corp., Armonk, N.Y., USA) and a two tailed $p$-value of $<0.05$ was considered statistically significant. Follow-up was presented as person-years from baseline to the date of death, loss to follow-up, or December 31, 2015. We built 3 Cox proportional regression models to assess the association between degree of daytime sleepiness (no daytime sleepiness, mild daytime sleepiness or EDS, with no daytime sleepiness as reference) and cardiovascular mortality. First (Model 1), we adjusted for socioeconomic factors including age, sex, race, family income, and education. Then we further adjusted for comorbidities and cardiovascular risk factors including alcohol, smoking, BMI $\left(\mathrm{kg} / \mathrm{m}^{2}\right)$, hypercholesterolemia, hypertension, diabetes mellitus, cardiovascular diseases, depression, COPD, cancer, and chronic kidney diseases in Model 2. Finally, in Model 3, we further adjusted for sleep duration and sleep disorders.

To test the robustness of our results and particularly the possibility of reverse causation, we performed sensitivity analyses by excluding participants who died within six months from baseline interview and by analyzing participants with or without cardiovascular disease at baseline. Furthermore, we investigated whether the relationship between EDS and cardiovascular mortality varied by age, sex, and BMI.

\section{Results}

\section{Baseline Characteristics}

Baseline characteristics of study population are presented in Table 1. Weighted mean age was 47.3 years $(95 \%$ CI, 46.0 to 48.1 years) and the majority of participants were non-Hispanic White (71.1\%). EDS was reported by $18.5 \%$ of participants, while $51.1 \%$ reported mild daytime sleepiness and $30.4 \%$ reported no daytime sleepiness. Compared 
Table I Baseline Characteristics by Baseline Daytime Sleepiness Category Among 10,330 Adults in the NHANES 2005-2008 ${ }^{\mathrm{a}}$

\begin{tabular}{|c|c|c|c|c|}
\hline \multirow[t]{2}{*}{ Characteristics } & \multicolumn{3}{|c|}{ Degree of Daytime Sleepiness ${ }^{e}$} & \multirow[t]{2}{*}{$P$-value } \\
\hline & No Daytime Sleepiness & Mild Daytime Sleepiness & EDS & \\
\hline Participants & 3878 & 4663 & 1789 & \\
\hline Age, years ${ }^{b}$ & $49.6(48.0,51.2)$ & $46.0(45.0,47.0)$ & $45.6(44.0,47.2)$ & $<0.0001$ \\
\hline $\begin{array}{l}\text { Age group }{ }^{b} \\
\leq 65 \text { years } \\
>65 \text { years }\end{array}$ & $\begin{array}{l}269 \mid(77.8) \\
\text { II87 (22.2) }\end{array}$ & $\begin{array}{l}3669(87.0) \\
994(13.0)\end{array}$ & $\begin{array}{l}1390(84.3) \\
399(15.7)\end{array}$ & $<0.0001$ \\
\hline $\begin{array}{l}\text { Sex }{ }^{b} \\
\text { Female } \\
\text { Male }\end{array}$ & $\begin{array}{l}1692(47.1) \\
2186(52.9)\end{array}$ & $\begin{array}{l}2349(51.0) \\
2314(49.0)\end{array}$ & $\begin{array}{l}1009(57.2) \\
780(42.8)\end{array}$ & 0.001 \\
\hline $\begin{array}{l}\text { Race } \\
\text { Hispanic } \\
\text { Non-Hispanic Black } \\
\text { Non-Hispanic White } \\
\text { Other }\end{array}$ & $\begin{array}{l}1249(18.1) \\
92 \mid(12.3) \\
1562(64.0) \\
146(5.6)\end{array}$ & $\begin{array}{l}1038(10.0) \\
982(10.4) \\
2454(73.9) \\
189(5.8)\end{array}$ & $\begin{array}{l}360(8.6) \\
354(10.6) \\
996(75.0) \\
79(5.6)\end{array}$ & $<0.0001$ \\
\hline BMI, $\mathrm{kg} / \mathrm{m}^{2}$ & $28.2(27.8,28.5)$ & $28.5(28.1,29.0)$ & $29.2(28.6,29.8)$ & 0.005 \\
\hline $\begin{array}{l}\text { Annual family income } \\
\quad<20,000 \\
\geq 20,000\end{array}$ & $\begin{array}{l}980(18.5) \\
2708(81.5)\end{array}$ & $\begin{array}{l}1010(13.8) \\
3521(86.2)\end{array}$ & $\begin{array}{l}530(22.2) \\
1188(77.8)\end{array}$ & $<0.0001$ \\
\hline $\begin{array}{l}\text { Education }^{\mathrm{b}} \\
\text { Less than college } \\
\text { College or above }\end{array}$ & $\begin{array}{l}2357(47.9) \\
1516(52.1)\end{array}$ & $\begin{array}{l}2263(39.7) \\
2,96(60.3)\end{array}$ & $\begin{array}{l}987(48.5) \\
799(51.5)\end{array}$ & 0.002 \\
\hline $\begin{array}{l}\text { Smoking status } \\
\text { Current } \\
\text { Former } \\
\text { Never }\end{array}$ & $\begin{array}{l}783(17.8) \\
1017(27.6) \\
2078(54.6)\end{array}$ & $\begin{array}{l}1066(24.0) \\
1158(23.8) \\
2430(52.2)\end{array}$ & $\begin{array}{l}482(31.4) \\
453(23.8) \\
853(44.8)\end{array}$ & $<0.0001$ \\
\hline $\begin{array}{l}\text { Alcohol consumption }{ }^{b} \\
\text { Yes } \\
\text { No }\end{array}$ & $\begin{array}{l}229 \mid(71.5) \\
105 \mid(28.5)\end{array}$ & $\begin{array}{l}3052(78.3) \\
1166(21.7)\end{array}$ & $\begin{array}{l}1074(70.6) \\
508(29.4)\end{array}$ & 0.0001 \\
\hline Hypertension $^{c}$ & $1652(35.0)$ & $1938(35.8)$ & $807(40.1)$ & 0.16 \\
\hline Diabetes $^{c}$ & $691(12.7)$ & $655(10.9)$ & $349(14.5)$ & 0.06 \\
\hline Hypercholesterolemia $^{c}$ & $1394(36.5)$ & $1779(36.9)$ & $739(37.6)$ & 0.91 \\
\hline Cardiovascular disease ${ }^{b}$ & $435(8.9)$ & $522(8.4)$ & $311(12.3)$ & 0.01 \\
\hline Chronic obstructive pulmonary disease ${ }^{b}$ & $209(5.2)$ & $358(6.5)$ & $246(13.7)$ & $<0.0001$ \\
\hline Cancer ${ }^{b}$ & $373(9.5)$ & $406(8.0)$ & $200(9.8)$ & 0.49 \\
\hline Chronic kidney disease ${ }^{c}$ & $432(7.9)$ & $433(6.3)$ & $231(8.8)$ & 0.09 \\
\hline Depression ${ }^{d}$ & $110(2.1)$ & $269(4.3)$ & $360(18.4)$ & $<0.0001$ \\
\hline $\begin{array}{l}\text { Sleep disorders } \\
\text { Sleep apnea } \\
\text { Insomnia } \\
\text { Restless legs } \\
\text { Other }\end{array}$ & $\begin{array}{l}162(4.0) \\
41(2.6) \\
3(0.2) \\
2(0.1) \\
116(1.1)\end{array}$ & $\begin{array}{l}318(6.9) \\
83(4.5) \\
13(0.6) \\
4(0.2) \\
218(1.6)\end{array}$ & $\begin{array}{l}312(15.4) \\
65(8.8) \\
11(1.5) \\
5(0.6) \\
231(4.5)\end{array}$ & $<0.0001$ \\
\hline
\end{tabular}


Table I (Continued).

\begin{tabular}{|l|l|l|l|l|}
\hline \multirow{2}{*}{ Characteristics } & \multicolumn{3}{|c|}{ Degree of Daytime Sleepiness } & \multirow{2}{*}{$P$-value } \\
\cline { 2 - 5 } & No Daytime Sleepiness & Mild Daytime Sleepiness & EDS \\
\hline Sleep duration, $^{\text {b }}$ hours & $7.1(7.0,7.2)$ & $6.9(6.8,7.0)$ & $6.3(6.1,6.4)$ & $<0.0001$ \\
\hline $\begin{array}{l}\text { Sleep duration b } \\
\leq 6\end{array}$ & $1166(29.0)$ & & $1864(36.9)$ & $1055(55.2)$ \\
$7-8$ & $2335(62.3)$ & $2498(56.8)$ & $617(39.3)$ \\
$\geq 9$ & $373(8.6)$ & $299(6.3)$ & $114(5.5)$ & $<0.0001$ \\
\hline
\end{tabular}

Notes: ${ }^{a}$ All estimates accounted for complex survey design. Data were presented as number $(\%)$ or means $(95 \% \mathrm{Cl}) .{ }^{\mathrm{b}}$ Variables were acquired through corresponding questionnaires. Sleep disorders included self-reported sleep apnea, insomnia, restless legs and other sleep disorders. ${ }^{\mathrm{C}}$ Variables were ascertained by both questionnaires and lab tests /examination. ${ }^{d}$ Depression was assessed using the Patient Health Questionnaire 9-item survey with a score $\geq 10$ indicative of major depression. ${ }^{\mathrm{e}}$ No daytime sleepiness was defined as never feeling overly sleepy during the day for last month; Mild daytime sleepiness was defined as rarely or sometimes (I to 4 times a month) feeling overly sleepy during the day; Excessive daytime sleepiness was defined as often or almost always (5 to 30 times a month) feeling overly sleepy during the day.

Abbreviations: BMI, body mass index. EDS, excessive daytime sleepiness.

with participants reporting no daytime sleepiness, those reporting EDS were slightly younger, more likely to be female, and non-Hispanic White. EDS participants had higher BMI and were more likely to have lower income, cardiovascular disease, COPD, and depression. Prevalence of sleep disorders and short sleep duration was also greater among those with EDS (Table 1).

\section{Association Between EDS and Cardiovascular Mortality}

During a mean follow-up of 8.3 years $(85,673$ person-years), a total of 262 cardiovascular deaths occurred. The crude cardiovascular mortality rate was 3.06 per 1000 person-years.

Compared with participants without daytime sleepiness, participants with EDS exhibited significantly increased risk of cardiovascular death (HR, 2.53, 95\% CI, 1.27 to 5.03) in multivariable analysis controlled for sociodemographic factors (Model 1, Table 2). The association between EDS and cardiovascular death was not attenuated by additional adjustment for baseline comorbidities and cardiovascular risk factors (Model 2, HR, 2.85, 95\% CI, 1.33 to 6.09). Further correction for sleep duration and sleep disorders (Model 3,
HR, 2.55, 95\% CI, 1.23 to 5.27) weakened the association only marginally (Figure 1 and Table 2).

\section{Subgroup and Sensitivity Analyses}

To examine whether the association between EDS and cardiovascular mortality varies based on demographic characteristics, we conducted subgroup analyses according to baseline age ( $>65$ years vs $\geq 65$ years), sex (male vs female) and BMI ( $\geq 30 \mathrm{~kg} / \mathrm{m}^{2}$ vs $\left.<30 \mathrm{~kg} / \mathrm{m}^{2}\right)$. Similar results were observed in these subgroups in the fully adjusted model 3 (Figure 2) and no interactions between EDS and age, sex or BMI were observed ( $P$ for interaction $>0.05$ ). However, men with EDS tended to have higher risk of cardiovascular mortality than women.

Exclusion of participants who died within six months from baseline interview did not diminish the strength of the association between EDS and cardiovascular death (HR, 2.45, 95\% CI, 1.20 to 5.00). The relationship between EDS and cardiovascular mortality was evident in participants without cardiovascular disease at baseline (HR, 2.34, 95\% CI, 0.82 to 6.67), and similar to those with cardiovascular disease at baseline (HR, 2.57, 95\% CI, 0.81

Table 2 Hazard Ratios (95\% Cls) of Cardiovascular Mortality with Daytime Sleepiness in NHANES 2005-2008 ${ }^{\mathrm{a}}$

\begin{tabular}{|l|l|l|l|l|}
\hline Variables $^{\text {b }}$ & No. (\%) of Events & Model I & Model 2 & Model 3 \\
\hline No DS & III (I.4) & Reference & Reference & Reference \\
Mild DS & $96(1.2)$ & $1.23(0.76,1.98)$ & $1.50(0.83,2.69)$ & $1.37(0.73,2.58)$ \\
EDS & $55(2.3)$ & $2.53(1.27,5.03)$ & $2.85(1.33,6.09)$ & $2.55(1.23,5.27)$ \\
\hline
\end{tabular}

Notes: a Model estimates were adjusted for the complex sampling design. Model I was adjusted for age, sex, race, education, and income. Model 2 was further adjusted for alcohol, smoking, body mass index, hypertension, hypercholesterolemia, cardiovascular disease, diabetes mellitus, depression, chronic obstructive pulmonary disease, chronic kidney disease and cancer. Model 3 was adjusted for sleep disorders and sleep duration in addition to the variables in model 2 . ${ }^{b} \mathrm{No}$ daytime sleepiness was defined as never feeling overly sleepy during the day for last month; Mild daytime sleepiness was defined as rarely or sometimes (I to 4 times a month) feeling overly sleepy during the day; Excessive daytime sleepiness was defined as often or almost always ( 5 to 30 times a month) feeling overly sleepy during the day. Abbreviations: DS, daytime sleepiness; EDS, excessive daytime sleepiness. 


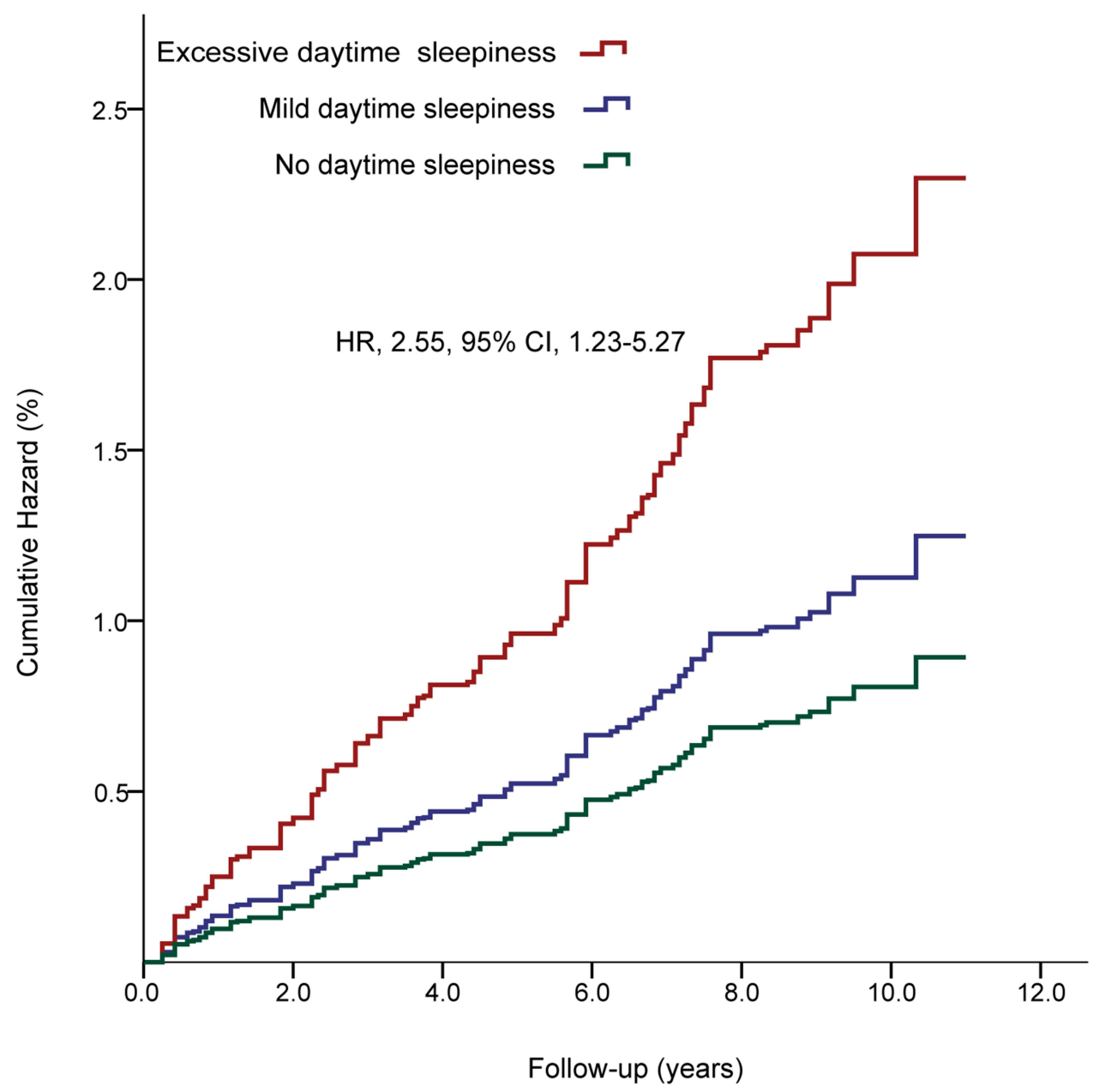

Figure I Cox cumulative hazard function for cardiovascular mortality by degree of daytime sleepiness. The model was fully adjusted for socioeconomic factors, cardiovascular risk factors, comorbidities, sleep duration and sleep disorders. HR, hazard ratio; No daytime sleepiness was defined as never feeling overly sleepy during the day for last month; Mild daytime sleepiness was defined as rarely or sometimes ( 1 to 4 times a month) feeling overly sleepy during the day; Excessive daytime sleepiness was defined as often or almost always (5 to 30 times a month) feeling overly sleepy during the day.

Abbreviation: $\mathrm{Cl}$, confidence interval.

to 8.15 ), but did not reach statistical significance likely due to the relatively low number of events (Table 3 ).

\section{Discussion}

In this prospective study of a representative sample of US adults, as far as we know, we first found that EDS was independently associated with an approximately two-and -a-half fold increased risk of cardiovascular mortality compared to absence of daytime sleepiness even after adjusting for sociodemographic factors, comorbidities, cardiovascular risk factors, sleep duration and sleep disorders. No interaction between EDS and age, sex or BMI was observed, but males with EDS tended to have higher risk of cardiovascular mortality than females. Additionally, we found that the prevalence of EDS was quite high with nearly one in five $(18.5 \%)$ adults reporting EDS in NHANES 2005-2008.

\section{Comparison with Other Studies}

The relationship between EDS and cardiovascular mortality has been rarely studied and current evidence is conflicting. To our knowledge, only a few prospective studies have assessed the relationship between EDS and cardiovascular mortality. The Three City Study ${ }^{20}$ defined EDS as self-reported regular or frequent feeling of being excessively sleepy during the day and found that EDS was associated with significantly increased risk of cardiovascular death. However, that study only assessed the association in elderly participants ( $\geq 65$ years) while we included both younger and older adults (all subjects $\geq 20$ years) from a nationally representative sample and observed no 


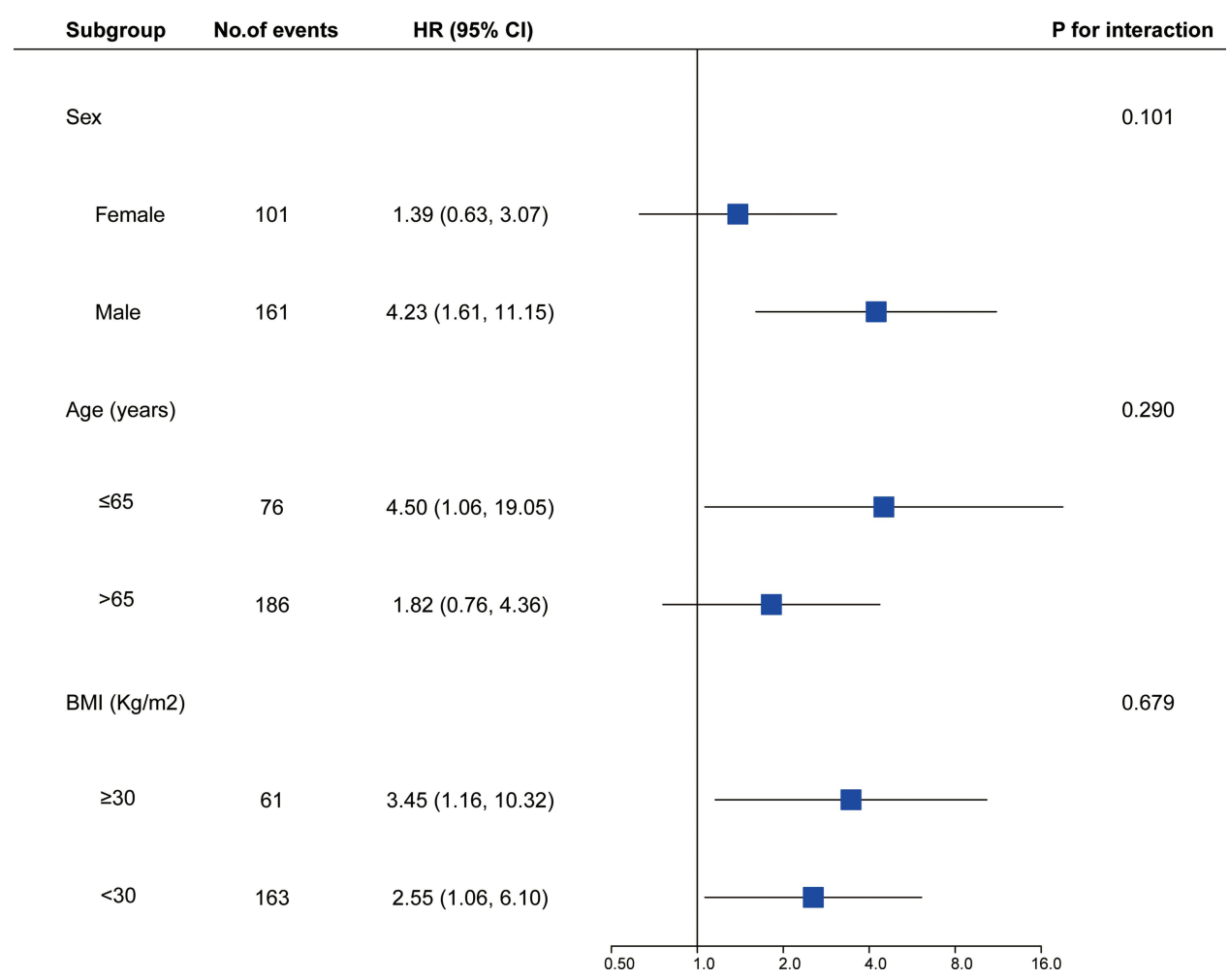

Figure 2 Associations between EDS and risk of cardiovascular mortality stratified by sex, age and body mass index in fully adjusted model. Abbreviations: BMI, body mass index; Cl, confidence interval; CVD, cardiovascular disease; EDS, excessive daytime sleepiness; HR, hazard ratio.

interaction between EDS and age $(P$ for interaction $=$ 0.290). Moreover, the Three City Study ${ }^{20}$ did not adjust for sleep apnea, restless legs or sleep duration, which have been reported as important risk factors for cardiovascular mortality. ${ }^{28-31}$ Lindberg et $\mathrm{al}^{21}$ conducted a prospective study enrolling 3100 male participants aged 30-69 years using a postal survey. After a 10-year follow-up, they reported no association between EDS and cardiovascular mortality. Nevertheless, the significantly higher mortality rate observed by the authors in the non-responders suggested that findings of this study were likely to be biased. In addition, Lindberg et al only controlled for age in their analysis. After analyzing the Northern Manhattan Study (NOMAS) data without adjusting for sleep disorders, Boden-Albala et al ${ }^{13}$ reported that EDS was significantly associated with stroke but not cardiovascular mortality in the general population aged 30-69 years. However, the small sample size of 2088 participants and shorter followup duration (5.1 years) in NOMAS might be the reason for the non-significant association between EDS and cardiovascular mortality, as the hazard ratio for cardiovascular mortality was 1.91 (95\% CI, 0.98 to 3.73 ). A metaanalysis pooling data from the three studies showed EDS was associated with significantly increased risk of

Table 3 Sensitivity Analyses of Association Between Daytime Sleepiness and Cardiovascular Mortality in Weighted and Fully Adjusted Multivariable Analysis (HR [95\% Cl])

\begin{tabular}{|l|l|l|l|}
\hline Variables $^{\mathbf{a}}$ & Exclusion of Deaths Within 6-Months & Without CVD at Baseline & With CVD at Baseline \\
\hline No. of events/total & $25 \mathrm{I} / \mathrm{I027I}$ & $141 / 9062$ & $12 \mathrm{I} / 1268$ \\
No DS & Reference & Reference & Reference \\
Mild DS & $1.1 \mathrm{I}(0.60,2.04)$ & $1.56(0.77,3.15)$ & $1.09(0.39,3.05)$ \\
EDS & $2.45(1.20,5.00)$ & $2.34(0.82,6.67)$ & $2.57(0.81,8.15)$ \\
\hline
\end{tabular}

Notes: ${ }^{a}$ No daytime sleepiness was defined as never feeling overly sleepy during the day for last month; Mild daytime sleepiness was defined as rarely or sometimes (I to 4 times a month) feeling overly sleepy during the day; Excessive daytime sleepiness was defined as often or almost always (5 to 30 times a month) feeling overly sleepy during the day.

Abbreviations: CVD, cardiovascular diseases; DS, daytime sleepiness; EDS, excessive daytime sleepiness. 
cardiovascular mortality. ${ }^{19}$ However, as the three original studies included in the meta-analysis did not properly adjust for the potential confounders (especially sleep apnea and sleep duration), results of this meta-analysis were not conclusive. The present study, using a large nationally representative sample and adjusting for established cardiovascular risk factors including comorbid sleep disorders, provides robust evidence for EDS as a risk factor for cardiovascular mortality in the general adult population. Supporting our finding, a cohort study with 0.5 million adults showed that self-reported difficulty in keeping sober-minded during the daytime was associated with increased risk of incident cardiovascular disease even after adjustment for multiple potential confounders including symptoms of early morning awakening and difficulties in initiating or maintaining sleep. ${ }^{32}$ Moreover, EDS has also been associated with $23 \%$ increase of all-cause mortality in a recent meta-analysis. ${ }^{19}$ Additionally, similar to the association between EDS and all-cause mortality, ${ }^{33}$ the association between EDS and cardiovascular mortality appeared stronger in males than females although no interaction between sex and EDS was observed.

\section{Potential Mechanisms}

Mechanisms that underlie the association between EDS and cardiovascular mortality remain poorly understood. EDS could be an early sign of developing cardiovascular or metabolic disease as multiple studies have reported association of EDS with cardiovascular diseases ${ }^{13-19,34}$ and obesity or diabetes. ${ }^{2,10}$ Results of a recent genome-wide association analysis (GWAS) illustrating significant positive genetic correlations between EDS and coronary heart disease and obesity provides further support for this hypothesis. ${ }^{35}$ In the same GWAS study, using Mendelian Randomization analysis, the authors also reported a potential causal association of higher BMI and diabetes with excessive daytime sleepiness. ${ }^{35}$ Chronic low-grade inflammation, an established mechanism for cardiovascular disease, ${ }^{36,37}$ obesity and diabetes, ${ }^{38,39}$ could be the key biological mechanism of these observed associations between EDS and cardiometabolic morbidity and mortality. Indeed, many studies have shown transient increase in subjective sleepiness following endotoxin administration ${ }^{40,41}$ and the association of sleepiness with inflammation among patients with sleep apnea $^{42,43}$ also provide as evidence for the inflammation hypothesis. However, considering scant evidence, further studies are needed to explore potential mechanism of the observed association.

\section{Clinical Implications}

Findings of the present study emphasize the importance of screening EDS in risk stratification and daily clinical practice, as we found that EDS was independently associated with an approximately two-and-a-half fold increased risk of cardiovascular mortality in a nationally representative cohort, a stronger predictor of cardiovascular death than some long established risk factors such as hypertension, ${ }^{44,45}$ obesity, ${ }^{46,47}$ and sedentary lifestyle. ${ }^{48,49}$ Therefore, screening for EDS could help identify patients at high risk of cardiovascular mortality and thus provide potential opportunity for early interventions in the clinical setting. Since screening for EDS is simple and costeffective compared with common cardiovascular disease biomarkers and may describe cardiovascular risk beyond traditional risk factors, EDS screening holds promise for improving identification of high-risk patients or underlying disorders. Furthermore, the association with increased risk of all-cause mortality demonstrated in a meta-analysis ${ }^{19}$ and the high prevalence of EDS observed in the present study and in previous studies ${ }^{20,50,51}$ also argue in favor of EDS screening in daily clinical practice.

\section{Strengths and Limitations}

Strengths of the present study include a large and nationally representative sample, long follow-up duration, high follow-up rate and extensive adjustment for potential confounders including sleep disorders and sleep duration. The present study has several limitations as well. First, the cardiovascular mortality analyzed in the present study did not include deaths due to stroke as NHANES 20072008 did not provide such data. Thus, our findings may not be generalized for the association between EDS and cerebrovascular mortality. Second, we used self-reported daytime sleepiness with one question which may lead to some power loss due to potential misclassification. Nevertheless, a previous study has shown that single question on sleepiness was significantly associated with the well-established Epworth Sleep Scale (ESS) for assessing EDS and the diagnostic specificity could be as high as $0.99 .{ }^{52}$ Although multiple sleep latency test (MSLT) is an objective tool to assess daytime sleepiness, it is not very practical to use it in large epidemiological studies and therefore have not been widely used in epidemiological studies. Furthermore, the large and representative sample may have mitigated the potential effect of misclassification. Third, considering the fact that sleep apnea remains quite 
underdiagnosed, ${ }^{53}$ we cannot rule out potential confounding effect of sleep apnea although we have adjusted for baseline sleep disorders. However, a recent study has found that significant increase of cardiovascular mortality was only observed in sleep apnea patients with EDS, ${ }^{54}$ and sleep apnea patients with EDS are much less likely to be undiagnosed. Therefore, the potential confounding effect of sleep apnea may have compromised by this fact. Fourth, although we have extensively adjusted for potential confounders, we were unable to exclude any potential effect of napping on the observed association between daytime sleepiness and cardiovascular mortality, as data on napping was not collected in NHANES. Lastly, we could not preclude the possibility of unidentified confounders in the observed associations as the present study is an observational study by nature. Therefore, future study is needed to confirm our findings.

\section{Conclusion}

Excessive daytime sleepiness is highly prevalent among US adults and is independently associated with an approximately two-and-a-half fold increased risk of cardiovascular mortality in a large national sample. Screening for excessive daytime sleepiness may be a simple and costeffective tool for identifying individuals at high risk of cardiovascular death.

\section{Data Sharing Statement}

All data in this study are publicly available from the NHANES study website (https://wwwn.cdc.gov/nchs/ nhanes/ContinuousNhanes/Default.aspx?BeginYear=2005).

\section{Funding}

These studies were supported in part by a grant from Sleep Number Corporation to the Mayo Clinic. VKS is supported by NIH HL65176, HL134885 and HL134808. JB is supported by T32 HL-007111. EM is supported by T32 HL 105355. JGL is supported by the National Natural Science Foundation of China (No. 82004301). NC is supported by NIH HL134885 and HL134808, by a grant from Sleep Number Corporation to the Mayo Clinic and by the Mayo Clinic Marie Ingalls Research Career Development Award. The funders had no role in design and conduct of the study; collection, management, analysis, and interpretation of the data; preparation, review, or approval of the manuscript; and decision to submit the manuscript for publication.

\section{Disclosure}

VKS has served as a consultant for Respicardia, Baker Tilly, Bayer and Jazz Pharmaceuticals and serves on the Sleep Number Research Advisory Board. VKS also reports grants from Sleep Number, during the conduct of the study; personal fees from Bayer, personal fees from Respicardia, personal fees from Jazz Pharmaceuticals, and personal fees from Baker Tilly outside the submitted work. All other authors claimed no conflicts of interest.

\section{References}

1. Hayley AC, Williams LJ, Kennedy GA, Berk M, Brennan SL, Pasco JA. Prevalence of excessive daytime sleepiness in a sample of the Australian adult population. Sleep Med. 2014;15(3):348-354. doi:10.1016/j.sleep.2013.11.783

2. Bixler EO, Vgontzas AN, Lin HM, Calhoun SL, Vela-Bueno A, Kales A. Excessive daytime sleepiness in a general population sample: the role of sleep apnea, age, obesity, diabetes, and depression. $J$ Clin Endocrinol Metab. 2005;90(8):4510-4515. doi:10.1210/ jc. 2005-0035

3. Ford ES, Cunningham TJ, Giles WH, Croft JB. Trends in insomnia and excessive daytime sleepiness among U.S. adults from 2002 to 2012. Sleep Med. 2015;16(3):372-378. doi:10.1016/j. sleep.2014.12.008

4. Pallesen S, Nordhus IH, Omvik S, Sivertsen B, Tell GS, Bjorvatn B. Prevalence and risk factors of subjective sleepiness in the general adult population. Sleep. 2007;30(5):619-624. doi:10.1093/sleep/ 30.5.619

5. Drake C, Roehrs T, Breslau N, et al. The 10-year risk of verifed motor vehicle crashes in relation to physiologic sleepiness. Sleep. 2010;33(6):745-752. doi:10.1093/sleep/33.6.745

6. Melamed S, Oksenberg A. Excessive Daytime sleepiness and risk of occupational injuries in non-shift daytime workers. Sleep. 2002;25 (3):315-322. doi:10.1093/sleep/25.3.315

7. Waldman LT, Parthasarathy S, Villa KF, Bron M, Bujanover S, Brod M. Understanding the burden of illness of excessive daytime sleepiness associated with obstructive sleep apnea: a qualitative study. Health Qual Life Outcomes. 2020;18(1):14. doi:10.1186/ s12955-020-01382-4

8. Jester DJ, Lee S, Molinari V, Volicer L. Cognitive deficits in Parkinson's disease with excessive daytime sleepiness: a systematic review. Aging Ment Health. 2020;24(11):1769-1780. doi:10.1080/ 13607863.2019.1660852

9. Leger D, Stepnowsky C. The economic and societal burden of excessive daytime sleepiness in patients with obstructive sleep apnea. Sleep Med Rev. 2020;51:11. doi:10.1016/j. smrv.2020.101275

10. Maugeri A, Medina-Inojosa JR, Kunzova S, et al. Sleep duration and excessive daytime sleepiness are associated with obesity independent of diet and physical activity. Nutrients. 2018;10(9):1219. doi:10.3390/nu10091219

11. Gangwisch JE, Rexrode K, Forman JP, Mukamal K, Malaspina D, Feskanich D. Daytime sleepiness and risk of coronary heart disease and stroke: results from the Nurses' Health Study II. Sleep Med. 2014;15(7):782-788. doi:10.1016/j.sleep.2014.04.001

12. Endeshaw Y, Rice TB, Schwartz AV, et al. Snoring, daytime sleepiness, and incident cardiovascular disease in the health, aging, and body composition study. Sleep. 2013;36(11):1737-1745. doi:10.5665/ sleep. 3140 
13. Boden-Albala B, Roberts ET, Bazil C, et al. Daytime sleepiness and risk of stroke and vascular disease: findings from the Northern Manhattan Study (NOMAS). Circ Cardiovasc Qual Outcomes. 2012;5(4):500-507. doi:10.1161/circoutcomes.111.963801

14. Xie J, Sert Kuniyoshi FH, Covassin N, et al. Excessive daytime sleepiness independently predicts increased cardiovascular risk after myocardial infarction. J Am Heart Assoc. 2018;7(2). doi:10.1161/ JAHA.117.007221

15. Kendzerska T, Gershon AS, Hawker G, Leung RS, Tomlinson G. Obstructive sleep apnea and risk of cardiovascular events and all-cause mortality: a decade-long historical cohort study. PLoS Med. 2014;11(2):e1001599. doi:10.1371/journal.pmed.1001599

16. Blachier M, Dauvilliers Y, Jaussent I, et al. Excessive daytime sleepiness and vascular events: the Three City Study. Ann Neurol. 2012;71 (5):661-667. doi:10.1002/ana.22656

17. Elwood P, Hack M, Pickering J, Hughes J, Gallacher J. Sleep disturbance, stroke, and heart disease events: evidence from the Caerphilly cohort. J Epidemiol Community Health. 2006;60 (1):69-73. doi:10.1136/jech.2005.039057

18. Qureshi AI, Giles WH, Croft JB, Bliwise DL. Habitual sleep patterns and risk for stroke and coronary heart disease: a 10-year follow-up from NHANES I. Neurology. 1997;48(4):904-911. doi:10.1212/ wnl.48.4.904

19. Wang L, Liu Q, Heizhati M, Yao X, Luo Q, Li N. Association between excessive daytime sleepiness and risk of cardiovascular disease and all-cause mortality: a systematic review and meta-analysis of longitudinal cohort studies. J Am Med Dir Assoc. 2020;21(12):1979-1985. doi:10.1016/j.jamda.2020.05.023

20. Empana JP, Dauvilliers Y, Dartigues JF, et al. Excessive daytime sleepiness is an independent risk indicator for cardiovascular mortality in community-dwelling elderly: the three city study. Stroke. 2009;40(4):1219-1224. doi:10.1161/strokeaha.108.530824

21. Lindberg E, Janson C, Svärdsudd K, Gislason T, Hetta J, Boman G. Increased mortality among sleepy snorers: a prospective population based study. Thorax. 1998;53(8):631-637. doi:10.1136/thx.53.8.631

22. Zipf G, Chiappa M, Porter KS, Ostchega Y, Lewis BG, Dostal J. National health and nutrition examination survey: plan and operations, 1999-2010. Programs Collection Procedures. 2013;1 (56): $1-37$.

23. National Center for Health Statistics. 2015 Public-Use Linked Mortality Files. Available from: https://www.cdc.gov/nchs/datalinkage/mortality-public.htm. Accessed January 11, 2021.

24. Levey AS, Stevens LA, Schmid CH, et al. A new equation to estimate glomerular filtration rate. Ann Intern Med. 2009;150 (9):604-612. doi:10.7326/0003-4819-150-9-200905050-00006

25. Kroenke K, Spitzer RL, Williams JB. The PHQ-9: validity of a brief depression severity measure. J Gen Intern Med. 2001;16(9):606-613. doi:10.1046/j.1525-1497.2001.016009606.x

26. Centers for Disease Control and Prevention (CDC). The National Health and Nutrition Examination Survey Tutorials; 2020. Available from: https://wwwn.cdc.gov/nchs/nhanes/tutorials/default.aspx. Accessed January 20, 2021.

27. Rao JNK, Scott AJ. On Chi-Squared Tests for Multiway Contingency Tables with Cell Proportions Estimated from Survey Data. Ann Statist. 1984;12(1):46-60. doi:10.1214/aos/1176346391

28. Young T, Finn L, Peppard PE, et al. Sleep disordered breathing and mortality: eighteen-year follow-up of the Wisconsin sleep cohort. Sleep. 2008;31(8):1071-1078.

29. Li Y, Li Y, Winkelman JW, et al. Prospective study of restless legs syndrome and total and cardiovascular mortality among women. Neurology. 2018;90(2):e135-e141. doi:10.1212/ wnl.0000000000004814

30. Kronholm E, Laatikainen T, Peltonen M, Sippola R, Partonen T. Selfreported sleep duration, all-cause mortality, cardiovascular mortality and morbidity in Finland. Sleep Med. 2011;12(3):215-221. doi:10.1016/j.sleep.2010.07.021
31. Ferrie JE, Shipley MJ, Cappuccio FP, et al. A prospective study of change in sleep duration: associations with mortality in the Whitehall II cohort. Sleep. 2007;30(12):1659-1666. doi:10.1093/sleep/ 30.12 .1659

32. Zheng B, Yu C, Lv J, et al. Insomnia symptoms and risk of cardiovascular diseases among 0.5 million adults: a 10-year cohort. Neurology. 2019;93(23):e2110-e2120. doi:10.1212/ WNL.0000000000008581

33. Merlino G, Lorenzut S, Gigli GL, et al. Insomnia and daytime sleepiness predict 20-year mortality in older male adults: data from a population-based study. Sleep Med. 2020;73:202-207. doi:10.1016/ j.sleep.2020.06.025

34. Fan M, Sun D, Zhou T, et al. Sleep patterns, genetic susceptibility, and incident cardiovascular disease: a prospective study of 385292 UK biobank participants. Eur Heart J. 2019;41(11):1182-1189. doi:10.1093/eurheartj/ehz849

35. Wang H, Lane JM, Jones SE, et al. Genome-wide association analysis of self-reported daytime sleepiness identifies 42 loci that suggest biological subtypes. Nat Commun. 2019;10(1):3503. doi:10.1038/ s41467-019-11456-7

36. Ridker PM, Everett BM, Thuren T, et al. Antiinflammatory therapy with canakinumab for atherosclerotic disease. $N$ Engl $J$ Med. 2017;377(12):1119-1131. doi:10.1056/NEJMoa1707914

37. Nidorf SM, Fiolet ATL, Mosterd A, et al. Colchicine in patients with chronic coronary disease. $N$ Engl J Med. 2020;383(19):1838-1847. doi:10.1056/NEJMoa2021372

38. Wellen KE, Hotamisligil GS. Inflammation, stress, and diabetes. J Clin Invest. 2005;115(5):1111-1119. doi:10.1172/jci200525102

39. Shoelson SE, Lee J, Goldfine AB. Inflammation and insulin resistance. J Clin Invest. 2006;116(7):1793-1801. doi:10.1172/ jci29069

40. Lasselin J, Karshikoff B, Axelsson J, et al. Fatigue and sleepiness responses to experimental inflammation and exploratory analysis of the effect of baseline inflammation in healthy humans. Brain Behav Immun. 2020;83:309-314. doi:10.1016/j.bbi.2019.10.020

41. Pollmächer T, Schuld A, Kraus T, Haack M. Experimental immunomodulation, sleep, and sleepiness in humans. Ann N Y Acad Sci. 2000;917(1):488-499. doi:10.1111/j.1749-6632.2000. tb05413.x

42. Huang T, Goodman M, Li X, et al. C-reactive protein and risk of OSA in four us cohorts. Chest. 2021;159(6):2439-2448. doi:10.1016/ j.chest.2021.01.060

43. Vgontzas AN, Papanicolaou DA, Bixler EO, et al. Sleep apnea and daytime sleepiness and fatigue: relation to visceral obesity, insulin resistance, and hypercytokinemia. J Clin Endocrinol Metab. 2000;85 (3):1151-1158. doi:10.1210/jcem.85.3.6484

44. Yamagishi K, Sawachi S, Tamakoshi A, Iso H. Blood pressure levels and risk of cardiovascular disease mortality among Japanese men and women: the Japan Collaborative Cohort Study for Evaluation of Cancer Risk (JACC Study). J Hypertens. 2019;37(7):1366-1371. doi:10.1097/hjh.0000000000002073

45. van den Hoogen PCW, Feskens EJM, Nagelkerke NJD, Menotti A, Nissinen A, Kromhout D. The relation between blood pressure and mortality due to coronary heart disease among men in different parts of the world. $N$ Engl J Med. 2000;342(1):1-8. doi:10.1056/ nejm200001063420101

46. Jiang J, Ahn J, Huang WY, Hayes RB. Association of obesity with cardiovascular disease mortality in the PLCO trial. Prev Med. 2013;57(1):60-64. doi:10.1016/j.ypmed.2013.04.014

47. Chen C, Ye Y, Zhang Y, Pan XF, Pan A. Weight change across adulthood in relation to all cause and cause specific mortality: prospective cohort study. BMJ. 2019;367:15584. doi:10.1136/bmj.15584

48. Powell KE, Thompson PD, Caspersen CJ, Kendrick JS. Physical activity and the incidence of coronary heart disease. Annu Rev Public Health. 1987;8:253-287. doi:10.1146/annurev. pu.08.050187.001345 
49. Bellettiere J, LaMonte MJ, Evenson KR, et al. Sedentary behavior and cardiovascular disease in older women. Circulation. 2019;139 (8):1036-1046. doi:10.1161/CIRCULATIONAHA.118.035312

50. Vashum KP, McEvoy MA, Hancock SJ, et al. Prevalence of and associations with excessive daytime sleepiness in an Australian older population. Asia Pac J Public Health. 2015;27(2):NP2275NP2284. doi:10.1177/1010539513497783

51. Young TB. Epidemiology of daytime sleepiness: definitions, symptomatology, and prevalence. J Clin Psychiatry. 2004;65(Suppl 16):12-16.
52. Zallek SN, Redenius R, Fisk H, Murphy C, O’Neill E. A single question as a sleepiness screening tool. J Clin Sleep Med. 2008;4 (2):143-148. doi: $10.5664 /$ jcsm. 27131

53. American Sleep Apnea Association. Sleep Apnea Information for Clinicians; 2020. Available from: https://www.sleepapnea.org/learn/ sleep-apnea-information-clinicians. Accessed February 2, 2021.

54. Mazzotti DR, Keenan BT, Lim DC, Gottlieb DJ, Kim J, Pack AI. Symptom subtypes of obstructive sleep apnea predict incidence of cardiovascular outcomes. Am J Respir Crit Care Med. 2019;200 (4):493-506. doi:10.1164/rccm.201808-1509OC

\section{Publish your work in this journal}

Nature and Science of Sleep is an international, peer-reviewed, open access journal covering all aspects of sleep science and sleep medicine, including the neurophysiology and functions of sleep, the genetics of sleep, sleep and society, biological rhythms, dreaming, sleep disorders and therapy, and strategies to optimize healthy sleep.
The manuscript management system is completely online and includes a very quick and fair peer-review system, which is all easy to use. Visit http://www.dovepress.com/testimonials.php to read real quotes from published authors. 